\title{
Color Content based Video Retrieval using Block Truncation Coding with Different Color Spaces
}

\author{
Sudeep.D.Thepade, \\ PhD. \\ Dean $(R \& D)$ and \\ Professor \\ Computer Engineering \\ Pimpri-Chinchwad \\ College of Engineering
}

\author{
Krishnasagar \\ Subhedarpage \\ B.E.Student
}

\author{
Department of Computer \\ Engineering \\ Pimpri-Chinchwad \\ College of Engineering
}

\author{
Ankur.A.Mali \\ B.E.Student
}
Department of Computer
Engineering
Pimpri-Chinchwad
College of Engineering

\author{
Tushar.S.Vaidya \\ B.E.Student
}
Department of Computer
Engineering
Pimpri-Chinchwad
College of Engineering

\begin{abstract}
The volume of video data is increasing because of advanced technology in imaging communication. This tends to increase the popularity the videos. The conventional textual description which is based on text entered by user, so, suffers from user perception. Hence, content based video retrieval is more attractive than text based retrieval, as it gives result proximity to semantic view. Here, a novel colour content based video retrieval based on block truncation coding is proposed with the help of seven assorted colour spaces. Video database of 500 video samples spread across 10 categories is considered as a test bed for experimentation. Performance comparison is done with the help of cross-over point of average precision and average recall values of 500 query videos fired on the whole database. Experimental results have shown performance improvement for video retrieval in chromaticityluminance colour spaces as compared conventional colour space (RGB). The best performance is observed by Block Truncation Coding withKekre's LUV colour space followed by $\mathrm{YCbCr}$ colour space.
\end{abstract}

\section{General Terms}

Content Based Video Retrieval (CBVR), Feature Extraction, Color Spaces, Block Truncation Coding (BTC)

\section{Keywords}

Content Based Video Retrieval (CBVR), Feature Extraction, Color Spaces, Block Truncation Coding (BTC)

\section{INTRODUCTION}

The advancement in imaging technology holds a very high attraction towards the videos rather than still images. Videos are considered to be more expressive in human communication. Due to advanced digital technology, these videos can be created, stored, deleted, updated, and edited by the user. Also video is gaining more popularity among users on internet. The user wants any video to be searched and retrieved for relevant matches from huge video pool. Due to ease of technology numbers of videos are being generated and stored resulting into voluminous video databases.

In, conventional text-based video retrieval $[8,9]$, the query is processed on the textual properties of the videos in database. In this retrieval, the irrelevant videos are also searched in user's point of view. So, if the keywords related to video is stored and accordingly the query is processed, then, there is searching difficulty aroused due to more keywords stored regarding each video in database. Also, if user's perspective is considered then, each video will have different keywords will be again ambiguity in video retrieval in this technique. To overcome this lacuna content based video retrieval is proposed [10,11], where the video is described with the help of its contents as in color, texture or shape information of the video frames.

Here, the novel idea is proposed as content based video retrieval (CBVR) using block truncation coding (BTC) with earlier color spaces in content based image retrieval [4] . In this approach, the color content of each video is considered to form feature vector.

As, proposed, the feature vector for each video is generated using block truncation coding technique. 5 frames are extracted from each video sample (here every 5 frame is considered). For every frame, the pixels are divided into two clusters based on the threshold value as upper cluster (having pixels greater than threshold) and lower clusters (having values lesser than threshold). The averages of these upper and lower clusters are considered as elements of the feature vector.

\section{BLOCK TRUNCATION CODING AND COLOR SPACES 2.1 BLOCK TRUNCATION CODING (BTC)}

Block truncation coding is a process in which colour content can be extracted and described each video in the database [4]. In this, each colour component threshold is generated. This threshold value is used to compute upper and lower value of that colour component. So, we get vector total 6 values for each frame in the video. This gives total 30 values of a video as a feature vector.

\subsection{COLOR SPACES USED}

\subsubsection{KEKRE'S LUV COLOUR SPACE}

This needs the conversion of RGB to Kekre's LUV components. The RGB to KLUV conversion matrix given in equation 1 gives the $\mathrm{L}, \mathrm{U}, \mathrm{V}$ components of color frame for respective $\mathrm{R}, \mathrm{G}$, and $\mathrm{B}$ components. 


$$
\left[\begin{array}{c}
L \\
U \\
V
\end{array}\right]=\left[\begin{array}{ccc}
1 & 1 & 1 \\
-2 & 1 & 1 \\
0 & -1 & 1
\end{array}\right]\left[\begin{array}{l}
R \\
G \\
B
\end{array}\right]
$$

\subsubsection{YCbCr COLOUR SPACE}

To get $\mathrm{YCbCr}$ components we need the conversion of RGB to $\mathrm{YCbCr}$ components. The RGB to YCbCr conversion matrix given in equation 2 gives the $\mathrm{Y}, \mathrm{Cb}, \mathrm{Cr}$ components of color frame for respective R, G, and B components of color frame.

$$
\left[\begin{array}{c}
Y \\
C b \\
C r
\end{array}\right]=\left[\begin{array}{ccc}
0.2989 & 0.5866 & 0.1145 \\
-0.1688 & -0.3312 & 0.5 \\
0.5 & -0.4184 & -0.0816
\end{array}\right]\left[\begin{array}{l}
R \\
G \\
B
\end{array}\right]
$$

\subsubsection{YUV COLOUR SPACE}

The RGB to YUV conversion matrix given in equation 3 gives the $\mathrm{Y}, \mathrm{U}, \mathrm{V}$ components of color frame for respective $\mathrm{R}$, $\mathrm{G}$, and $\mathrm{B}$ components.

$$
\left[\begin{array}{l}
Y \\
U \\
V
\end{array}\right]=\left[\begin{array}{ccc}
0.299 & 0.587 & 0.144 \\
-0.14713 & -0.22472 & 0.436 \\
0.615 & -0.51498 & 0.10001
\end{array}\right]\left[\begin{array}{l}
R \\
G \\
B
\end{array}\right]
$$

\subsubsection{YIQ COLOUR SPACE}

To obtain YIQ color space is done by using equation 4 from RGB color space.

$$
\left[\begin{array}{l}
Y \\
I \\
Q
\end{array}\right]=\left[\begin{array}{ccc}
0.299 & 0.587 & 0.144 \\
0.595716 & -0.274453 & -0.32126 \\
0.211456 & -0.522591 & 0.31135
\end{array}\right]\left[\begin{array}{l}
R \\
G \\
B
\end{array}\right]
$$

\subsubsection{YCgCb COLOUR SPACE}

Interconversion equations for $\mathrm{RGB}$ to $\mathrm{YCgCb}$ color space is given in equation 5 .

$$
\left[\begin{array}{c}
Y \\
C g \\
C b
\end{array}\right]=\left[\begin{array}{ccc}
1 & 1 & 1 \\
1 & -1 & 0 \\
1 & 0 & -1
\end{array}\right]\left[\begin{array}{l}
R \\
G \\
B
\end{array}\right]
$$

\subsubsection{XYZ COLOUR SPACE}

Conversion equations for RGB to $\mathrm{XYZ}$ color space and $\mathrm{XYZ}$ to RGB is given in equation 6 below.

$$
\left[\begin{array}{c}
X \\
Y \\
Z
\end{array}\right]=\left[\begin{array}{ccc}
0.412453 & 0.357580 & 0.180423 \\
0.212671 & 0.71160 & 0.072169 \\
0.019334 & 0.119193 & 0.950227
\end{array}\right]\left[\begin{array}{c}
R \\
G \\
B
\end{array}\right]
$$

\section{CONTENT BASED VIDEO RETRIEVAL (CBVR)}

Content Based Video Retrieval relies on the characterization of primitive features such as colour, shape and texture that can be automatically extracted from the videos themselves.

Queries to CBVR system are most often expressed as colour feature vector of video and then similarity comparison is performed on query. And the most relevant videos are retrieved.

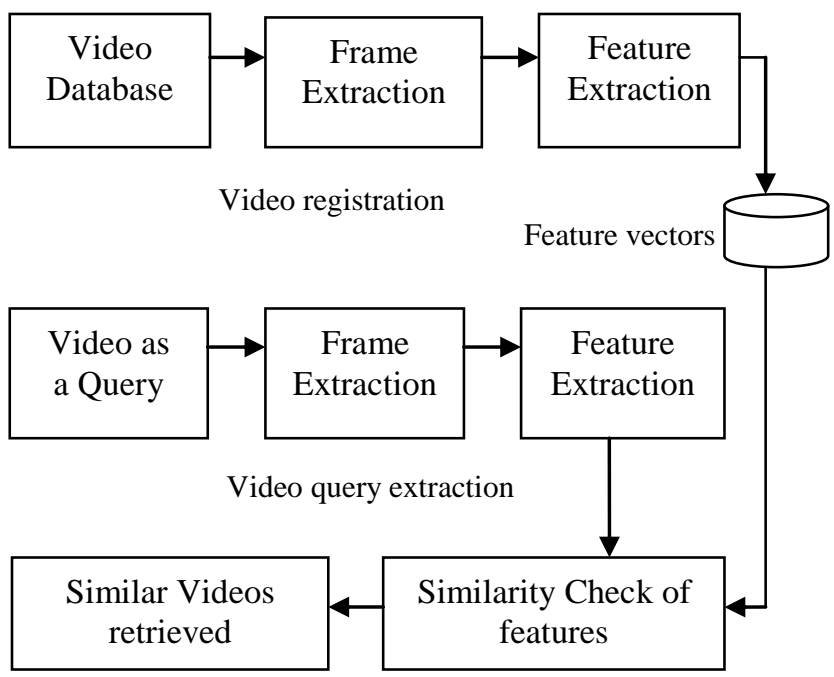

Fig 1 Flow of a typical CBVR process

\subsection{COLOR RETRIEVAL}

Several methods for retrieving videos on the basis of color similarity have been described in the literature, but most are variations of the same basic idea. Each video added to the collection is analyzed to compute a color feature vector, which shows the proportion of pixels of each color within the video. The color feature vector for each video is then stored in the database. Here BTC is applied on video frames to get the feature vectors

\section{IMPLEMENTATION}

\subsection{PLATFORM EXPERIMENTATION}

The implementation of model is done in MATLAB 2012a with basic system of Intel core 2 duo $(2.93 \mathrm{GHz})$ with $2 \mathrm{~GB}$ RAM and minimum of 250GB hard disk for storage. The modules of model are run under MATLAB 2012a compiler. The operating system used is windows 7 for mat lab environment.

\subsection{VIDEO DATASET}

The videos are categorized in 10 assorted categories containing 50 similar videos. So, in total there are 500 videos in data set for experimentation. The statistical details regarding da sed is given in table I. Figure 2shows the sample frames extracted from respective videos of each category. 

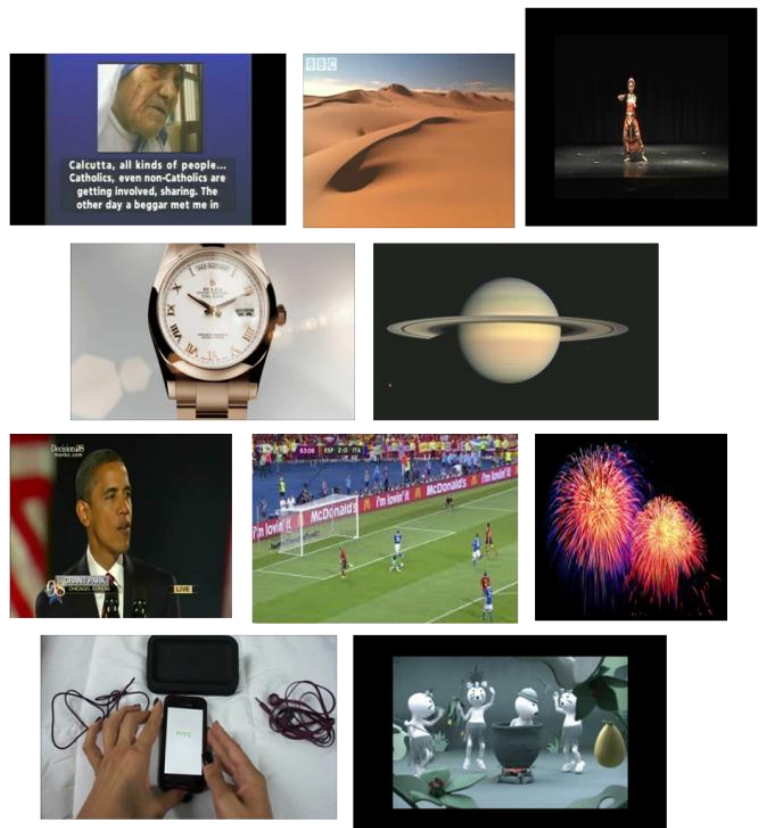

Fig 2: Example Frames from the Video Samples from Video database

TABLE I: Statistics of Video Dataset

\begin{tabular}{|l|c|}
\hline CATEGORIES & NUMBER OF VIDEOS \\
\hline OBAMA_ELECTION & 50 \\
\hline FIREWORKS & 50 \\
\hline GADGETS & 50 \\
\hline SOLAR SYSTEM & 50 \\
\hline DESERT & 50 \\
\hline MOTHER TERESSA & 50 \\
\hline DANCE & 50 \\
\hline ROLEX_WATCHES & 50 \\
\hline FOOTBALL & 50 \\
\hline ZOO-ZOO & \\
\hline
\end{tabular}

\subsection{FEATURE EXTRACTION}

In this, feature vectors are generated by using 5 frames of each video and by applying BTC on them in 7 different color spaces. Then, these feature vectors are used for query processing and performance comparison. The figure 3 shows color feature extraction using BTC

\subsection{PERFORMANCE COMPARISON PARAMETERS}

The performances of the proposed approaches are evaluated on the video dataset using the precision-recall cross over points. Precision-recall are given by equations 7 and 8 .

$$
\begin{aligned}
& \text { Recall }=\frac{\text { No of relevant items retrieved }}{\text { Total no of items retrieved }} \\
& \text { precision }=\frac{\text { No of Relevant items retrieved }}{\text { No of items retrieved in database }}
\end{aligned}
$$

\subsection{SIMILARITY MEASUREMENTS}

Here mean square error (MSE) and absolute difference (AD) are used for similarity measurements of feature vectors of the videos in content based video retrieval

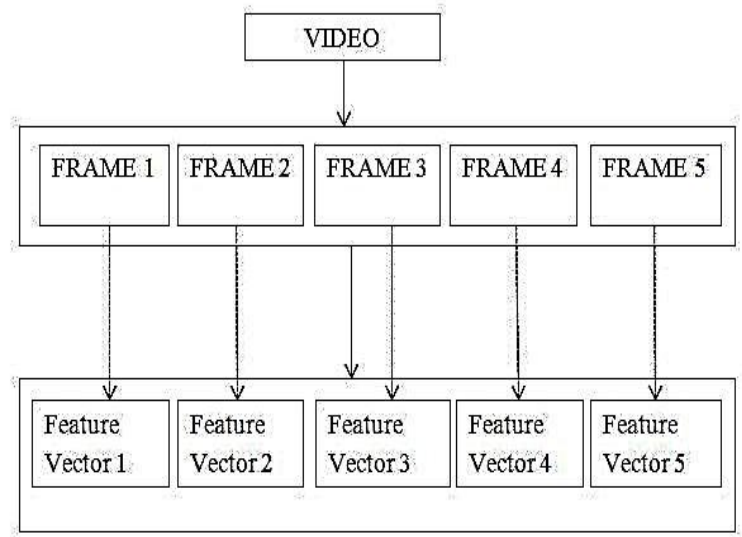

Fig 3 Feature Extraction Using BTC

\section{RESULTS ANS DISCUSSION}

In all 14 different variations of the proposed CBVR method are experimented with 500 queries fired on video database for each variation. 7 color spaces and two similarity measurement criteria are considered. The precision-recall cross over points of each query is computed to get the average precision-recall cross point of respective variation of proposed CBVR. Higher the cross over point value, better is the CBVR performance Figure 4 shows average cross-over points of precision-recall values for respective color spaces obtained using absolute difference in BTC based CBVR. Here, the Kekre's LUV color space out performs other color spaces for CBVR using BTC. Second best performance is observed by $\mathrm{YCbCr}$ color space. In fact, all luminance-chromaticity color spaces (YCgCb,KLUV, YIQ, YCbCr, and YUV) have shown better performance than conventional RGB color space.

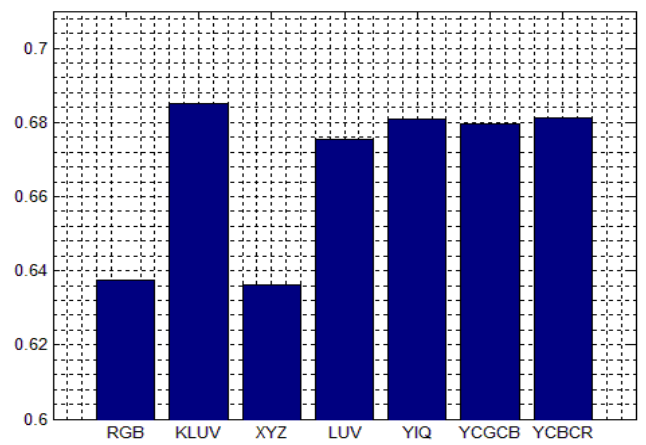

Fig 4: Average precision-recall cross over point values of BTC based CBVR using AD for respective color spaces. 


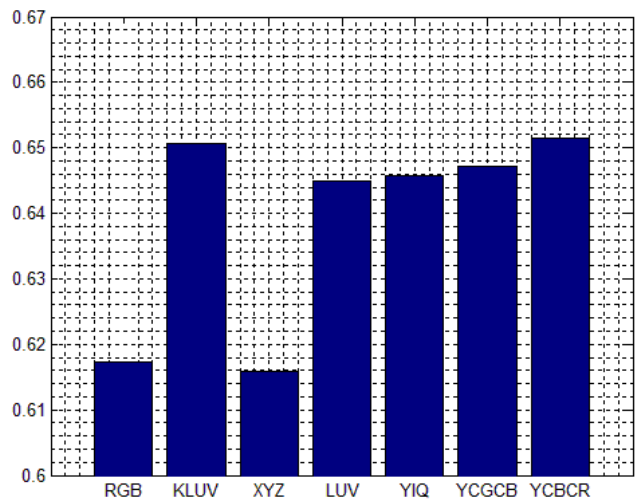

Fig 5: Average precision-recall cross over point values of BTC based CBVR using MSE for respective color spaces.

Figure 5 shows the average cross over points of precisionrecall values for respective color spaces obtained using MSE in BTC based CBVR. Also, in case of MSE the best performance is observed with Kekre's LUV color space.

\section{CONCLUSION}

It gives light on selection of feature extraction methods for BTC.Content based video retrieval is gaining momentum over text based video retrieval. Here, a novel color content based video retrieval approach is proposed using block truncation coding. In all, fourteen variations of the proposed CBVR methods are experimented using 7 different color spaces (RGB,XYZ, YCgCb,YCbCr,KLUV,YIQ,YUV) and two similarity measurement criteria (MSE,AD). The Kekre's LUV color space has shown best performance for BTC based CBVR among all considered color spaces. The performance ranking of color spaces is similar in BTC based CBVR for both the considered similarity measurement criteria. As computational complexity of absolute difference is lesser, it can be considered better over MSE. This experimentation helped in improvising ourunderstanding about efficiency of different color spaces while studying color contents of videos. BTC is proved to be better method used to extract color contents of videos and represent as a feature vector with Kekre's LUV also withabsolute difference as similarity measure.

\section{REFERENCES}

[1]. GuopingQiu, "Color Image Indexing Using BTC,"IEEE Transactions on Image Processing, VOL.12, NO.1, pp.93-101, January 2003.

[2]. H. B. Kekre, Sudeep D. Thepade, VarunLodha, PoojaLuthra, Ajoy Joseph,
ChitrangadaNemani,"Augmentation of Block Truncation Coding based Image Retrieval by using Even and Odd Images with Sundry Colour Spaces" H.B.Kekre et al. / (IJCSE) International Journal on Computer Science and Engineering Vol. 02, No. 08, 2010, 2535-2544

[3]. Dr.H.B.Kekre, Sudeep D. Thepade, "Boosting Block Truncation Coding using Kekre's LUV Color Space for Image Retrieval", WASET International Journal of Electrical, Computer and System Engineering (IJECSE), Volume 2, Number 3, pp. 172-180, Summer 2008. Available online at http://www.waset.org/ijecse/v2/v2-323.pdf

[4]. Dr.H.B.Kekre, Sudeep D. Thepade, "Image Retrieval using Augmented Block Truncation Coding Techniques", ACM International Conference on Advances in Computing, Communication and Control (ICAC3-2009), pp. 384-390, 23-24 Jan 2009, Fr. ConceicaoRodrigous College of Engg., Mumbai. Is uploaded on online ACM portal.

[5]. Dr.H.B.Kekre, Sudeep D. Thepade, "Using YUV Color Space to Hoist the Performance of Block Truncation Coding for Image Retrieval", IEEE International Advance Computing Conference 2009 (IACC ${ }^{\mathrm{e}}$ 09), Thapar University, Patiala, INDIA, 6-7 March 2009.

[6]. Dr.H.B.Kekre, Sudeep D. Thepade, "Color Based Image Retrieval using Amendment Block Truncation Coding with YCbCr Color Space", International Journal on Imaging (IJI), Volume 2, Number A09, Autumn 2009, pp. 2-14. Available online at www.ceser.res.in/iji.html (ISSN: 0974-0627)

[7]. M.Petkovic "Content-based video retrieval",Centre for Telematics and Information Technology, University of Twente.

[8]. Zhang, Hong Jiang, et al. "An integrated system for content-based video retrieval and browsing." Pattern recognition 30.4 (1997): 643-658.

[9]. Shim, Jae-Chang, ChitraDorai, and Ruud Bolle. "Automatic text extraction from video for content-based annotation and retrieval." Pattern Recognition, 1998. Proceedings. Fourteenth International Conference on. Vol. 1. IEEE, 1998.

[10].Zhang, Hong Jiang, Jianhua Wu, Di Zhong, and Stephen W. Smoliar. "An integrated system for content-based video retrieval and browsing." Pattern recognition 30, no. 4 (1997): 643-65

[11].Jain, Anil K., AdityaVailaya. "Image retrieval using color and shape."Pattern recognition 29.8 (1996):123312 